\title{
INDOOR 3D ROUTE MODELING BASED ON ESTATE SPATIAL DATA
}

\author{
Hongping Zhang ${ }^{\text {a }}$, Yongning Wen ${ }^{\text {b }}$, Jie Jiang ${ }^{\mathrm{b}}$, Wei Huang ${ }^{\mathrm{a}}$ \\ ${ }^{a}$ National Geomatics Center of China, Beijing, China-(zhanghongping, jjie, huangwei) @ ngcc.cn \\ ${ }^{\mathrm{b}}$ Key Laboratory of Virtual Geographic Environment, Ministry of Education, Nanjing Normal University, China-(wenyn@msn.com)
}

\author{
Commission IV, WG IV/7
}

KEY WORDS: Indoor three-dimensional route model, Axis Extraction, Route Planning

\begin{abstract}
:
Indoor three-dimensional route model is essential for space intelligence navigation and emergency evacuation. This paper is motivated by the need of constructing indoor route model automatically and as far as possible. By comparing existing building data sources, this paper firstly explained the reason why the estate spatial management data is chosen as the data source. Then, an applicable method of construction three-dimensional route model in a building is introduced by establishing the mapping relationship between geographic entities and their topological expression. This data model is a weighted graph consist of "node" and "path" to express the spatial relationship and topological structure of a building components. The whole process of modelling internal space of a building is addressed by two key steps: 1) each single floor route model is constructed, including path extraction of corridor using Delaunay triangulation algorithm with constrained edge, fusion of room nodes into the path; 2) the single floor route model is connected with stairs and elevators and the multi-floor route model is eventually generated. In order to validate the method in this paper, a shopping mall called "Longjiang New City Plaza" in Nanjing is chosen as a case of study. And the whole building space is constructed according to the modelling method above. By integrating of existing path finding algorithm, the usability of this modelling method is verified, which shows the indoor three-dimensional route modelling method based on estate spatial data in this paper can support indoor route planning and evacuation route design very well.
\end{abstract}

\section{INTRODUCTION}

With the rapid development of navigating technique and space technology, outdoor routing technology has got fully development and application, and vehicular navigation system and mobile navigation platform is playing important roles in people's daily life. Those who equipped with the devices are allowed to walk or travel around the complicated streets easily and effectively. However, it's not convenient to find out a special a particular place, person, or object in some complicated or unfamiliar indoor environment, especially for the first time. In addition, according to some statistics, people spend on over $80 \%$ of their time to work and live indoor. Because of complex internal structure, functions and dense people of modern city buildings, the frequency and severity of emergencies, such as a fire and harmful gas leakage, is increasing and brings heavy losses for individuals. When a building catches fire, it is difficult to evacuate from a smoke-filled building, even if you follow the emergency channel signs. As the death toll from the building fires is on rise year by year, which makes it the deadliest in various kinds of fires. Therefore, people are focusing now more than ever on intelligent indoor navigation and path planning for emergency, which is also as a part of the city emergency administration system (Huang, Y. 2010).

Some commercial indoor navigation systems have been introduced in the past few years, for instance, "Micello App" created by Google, and "Destination Maps" developed by Nokia. These applications are focus on indoor localization and maps, which provides solid technique basis for indoor navigation and emergency evacuation. However, due to the lack of modelling ways for indoor space and analysis of the characteristics of building data sources, the path planning algorithms cannot be used for emergency evolution easily (Stevens, M., 2006).
Therefore, this paper is motivated by the need of constructing indoor route model automatically and as far as possible. Threedimensional route model of a building is presented, with particular emphasis on its design procedures and characters, and the model is a weighted graph consist of nodes and edges. The construction process of the model is described in detail, including extracting hallway route, adding room nodes, and the whole solution for constructing the model. By integrating of existing path finding algorithm, the usability of this modelling method is verified.

\section{ESTATE SPATIAL DATA ORGANIZATION}

An effective management method for estate spatial data in China has been created through several decades of development, which is organized based on for basic levels "Region-BuildingFloor-Unit", as shown in Figure 1.

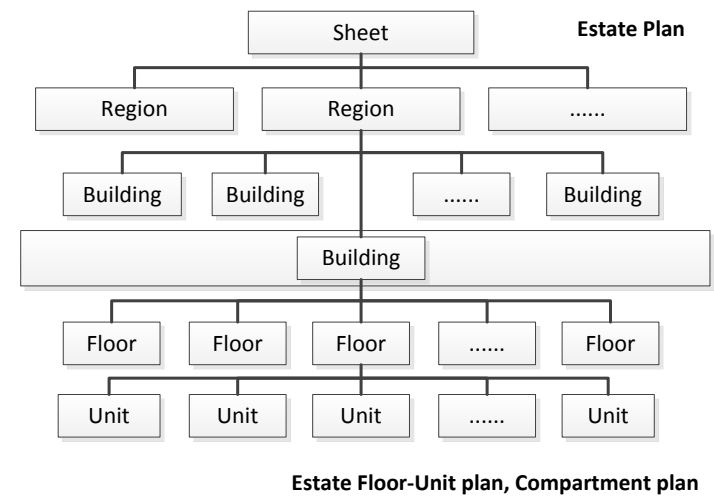

Figure 1 Framework of estate spatial data organization 
The estate floor-unit plan is referred to the details in a building, which is sheeted according to the floors. Each floor is the basic feature of the plan, and units are the logical entities for ownership management. Thus, the constitute features of estate floor-unit plan include compartments, wall centrelines, annotations and subsidiary facilities, including doors, windows, etc.

Based on the estate floor-unit plan, attached facilities are added, such as water-supply equipments, fire-fighting equipments, electricity-supply equipments and so on, which forms estate compartment plan. This provides great convenience for constructing a precise indoor route analysis model, and makes it possible to be used in indoor navigation, emergency rescue and evacuation route analysis. Figure 2 shows the estate floor-unit plan, compartment plan and their relationships.

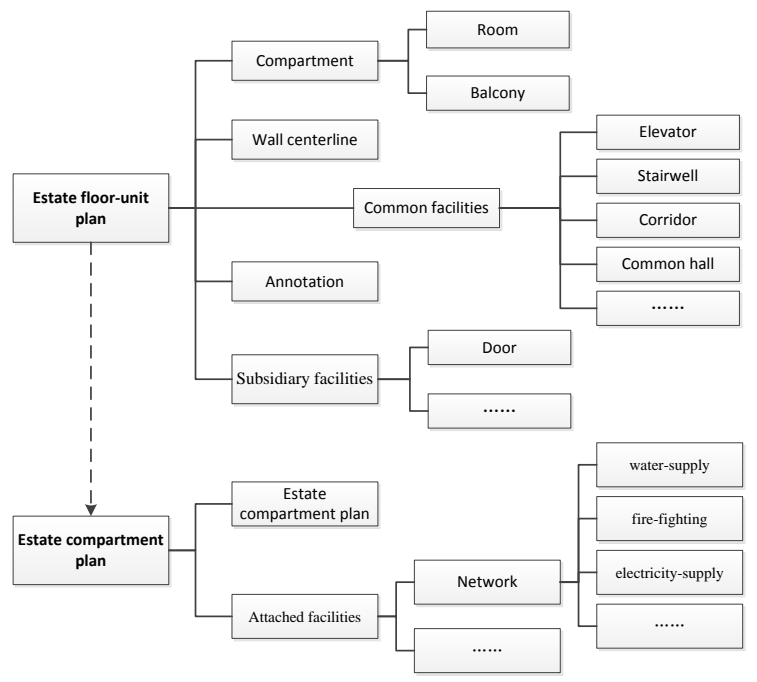

Figure 2 Estate floor-unit plan, compartment plan and their relationships

Due to the advantages of high accuracy of position, complete topological relationship, rich attribute information, and widespread use, the estate spatial data is selected as the major data source to research on 3D Routing modelling in a MultiLevel Building.

\section{INDOOR 3D ROUTE MODEL DESIGN}

The $3 \mathrm{~d}$ route model is proposed based on topological graph(Krieg Brückner, 2005), and its core is that the basic network for walking is composed of all connected paths in the building. More importantly, the geometry, semantic ,and topological information is included, which not only means spatial location, type, name, ownership, etc, but also the spatial relations such as adjacency or association, namely topological relations( Stoffel, E. P., 2007).

"Path" and "node" are the basic factors to illustrate the topological relations of the route network, in which "path" represents the connectivity between nodes and it is a directed line-segment. The topological structure of the whole route network in the building can be reflected by connecting all paths and nodes. Nodes, including endpoints of a path, crossing points, and junction points, are used to express the junction relations among paths, and can be shared by multiple path. The connectivity of nodes, expressed by paths, constructs indoor network structure .

The indoor $3 \mathrm{~d}$ route proposed in this paper is to establish relationships between entities and topological expression. Every compartment is represented with the door, and internal space is ignored. The method not only can ensure the accuracy of internal space abstraction, but also not influence the result of route planning. For compartments with internal rooms, it can be represented with abstract door and compartment model. The relationships among objects of the $3 \mathrm{~d}$ route model is described by using UML modeling language as shown in Figure 3.

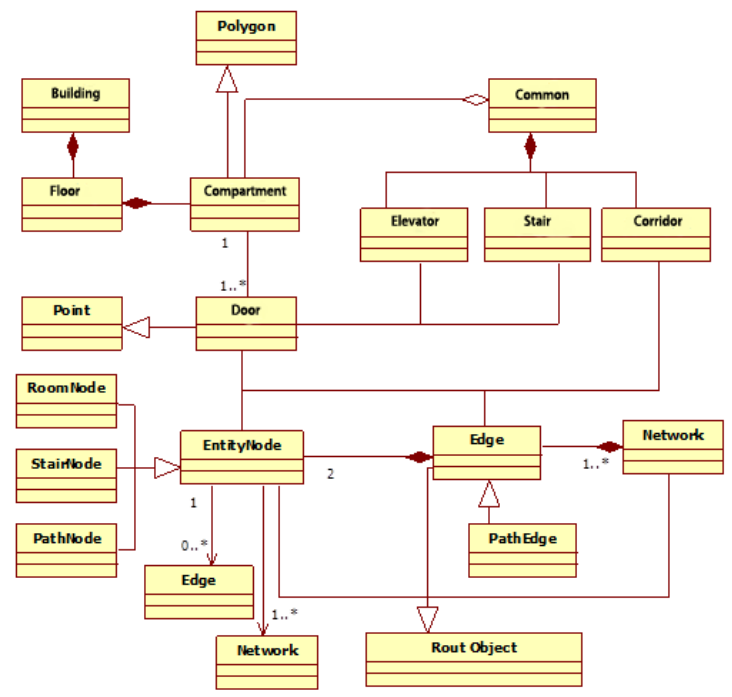

Figure 3 The UML for building space model

According to the analysis of estate spatial data, it can be found that the "floor" is the key physical space, which contains all based elements (compartments), connection elements(elevators and stairs ) between floors, and connection elements(corridor) among compartments. Therefore, the "floor" is the link between the building and compartments.

The semantic relationships is included in estate spatial data, for example, a building is composed of floors, and a door is located in a compartment. This kind of semantic relationships are reserved to guarantee the consistency and integrity in the in aspects of geometry, topology, semantics, etc.

All rooms are represented with nodes, and the doors are used to represented rooms, which are the critical associated objects to access to the compartments. As for the corridor area, it is represented by edges, and the crossing points and turning points are represented by nodes. The rout graph can be constructed by nodes and edges, and the weight of each edge is the traffic cost.

\section{INDOOR 3D ROUTE MODEL CONSTRUCTION}

The overall thought of constructing indoor $3 \mathrm{~d}$ route model automatically is mainly divided in to three steps as follows: (1) using nodes to represent compartments, such as rooms, stairwells, and using edges to represent the corridor area; (2) extracting the medial axis of the corridor and jointing compartments nodes to it, and then a single floor route model is created; (3) connecting stairs and elevators of two adjacent single-floor route models and the whole $3 \mathrm{D}$ route model is 
constructed. Figure 4 shows a specialty process of modelling, and the most important of which is to constructing the single floor route model.

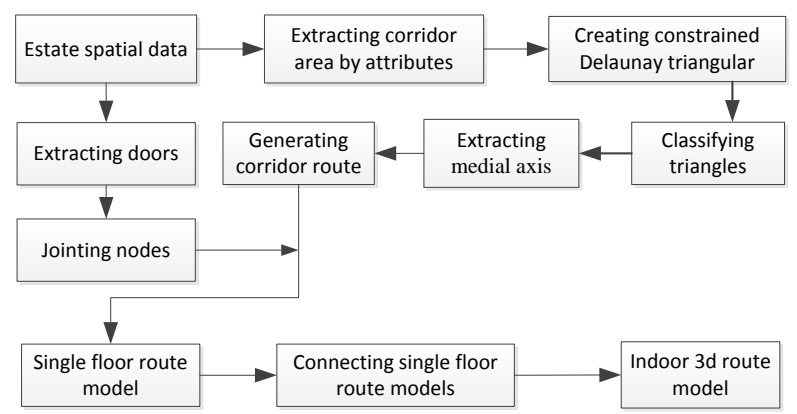

Figure 4 The process of building space modelling

An indoor $3 \mathrm{~d}$ route model is generated by connecting stairs and elevators of two adjacent single-floor route models, including path weights within it, which are used for route planning.

\subsection{Single floor route model construction}

4.1.1 Corridor route extraction: The corridor area data can be selected from estate compartment plan by filtering attributes, which is used for triangles generation.

4.1.2 Constrained Delaunay triangles generation : With the constraints of vertices of corridor area as operands, the constrained Delaunay triangles are generated, and the constraint conditions is that the triangle cannot exceed the boundary.

4.1.3 Triangles classification: According to the adjacency relationship among the edges of each triangle, that is the number of adjacent triangles, the triangles are grouped into three types: Type I, Type II and Type III, e.g. Figure 5.
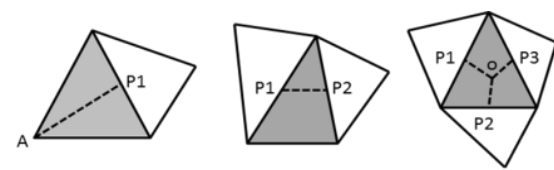

Figure 5 Classification of triangles

4.1.4 Medial axis extraction from triangulation: Because of the nearest neighbor (NN) algorithm of delaunay triangulation (Preparatat, F. P, 1985), the edge of a triangle can be seen as an adjacent connection between a point of the polygon boundary to other points of boundary. Thus, the midpoint of an common edge between triangles can be considered as an center of the polygon in the local are. The medial axis is constructed by connecting adjacent common midpoints of triangles, which illustrates the extended trend of the polygon from the perspective of one dimension. As shown in Figure 5, for Type I triangle, the vertex should be connected to the midpoint of one edge shared between adjacent triangles ( $A \rightarrow P 1$ or $P 1 \rightarrow A$ ); for Type II triangle, the midpoints of two edges shared between adjacent triangles $(P 1 \rightarrow P 2$ or $P 2 \rightarrow P 1)$; for Type III triangle, the triangle-center should be connected to the midpoints of three edges shared between adjacent triangles ( $O \rightarrow P_{i}$ or $\left.P_{i} \rightarrow O, \mathrm{i}=1,2,3\right)$. The algorithm of axis extraction is described as follows: (1) Two collections of stack are created, a traversal triangles collection $\{\mathrm{TRI}\}$ and branch triangles collection $\{\mathrm{BRANCH}\}$, which are used for record all traversed triangles and all traversed branch triangles(Type III) sequentially. The traversal can start from any triangle of type III, and this triangle should be pushed into $\{$ BRANCH $\}$, while extending to adjacent triangles along the directions of three edges. (2) If the current triangle type is II, it is indicated that the extending direction is unique and should be pushed into $\{\mathrm{TRI}\}$; (3) if the current triangle type is III, it is indicated that there are two extending directions and the current edge must be marked and continued to traverse along one edge. The extending direction of axis is determined by the triangle of Type III, and this triangle should be pushed into $\{\mathrm{BRANCH}\}$. (3) If the current triangle type is $\mathrm{I}$, it is the end of this branch, and this triangle should be pushed into \{TRI\}. (4) Repeat the above steps and check collection $\{$ BRANCH \} whether it is null. Get the top triangle of the stack and traverse along the edges without marks. If three edges of the triangle has been traversed, it should be popped from the stack. The axis of corridor is finished while the collection $\{$ BRANCH \} is null. Figure 6 shows the result of axis extraction.

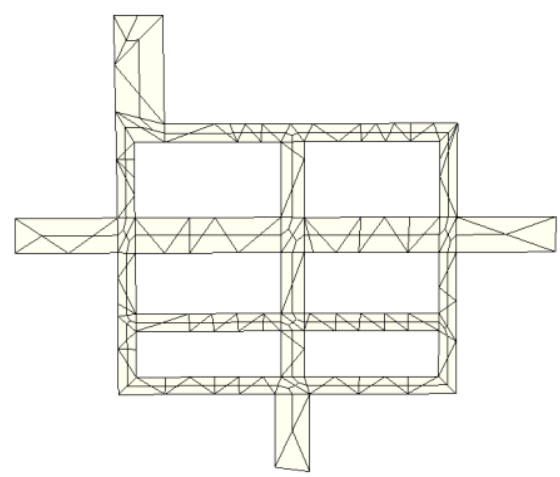

Figure 6 Axis extraction from delaunay triangulation for corridor modelling

4.1.5 Topological route construction: The axis is composed of many continuous points, and some may be separate linesegments. It is easy to generate edges with two endpoints of each line-segment. The conversion of thought is as follows: (1) Get a line-segment $(\mathrm{L})$ of a path and use "s" and "e" to represent its endpoints.(2) Check the route graph if the node is exist. (3) Add new nodes "PS" and "PE" with the position data of "s" and "e". (3) Add a new edge with the nodes of "PS" and "PE", and the initial weight is the length, then return to step1. Repeat the above operation and the topological route will be generated until all line-segments have been calculated.

4.1.6 Compartments connection: A complete single-floor route requires not only topological route, but also the compartments should be added to the route model. Generally, rooms are represented with central point. However, there may be more than one doors for a room, especially some business towers with complicated internal structures. The route solution calculated with room's central point may not be the most optimal in this case. Since doors are the connection between rooms and the corridor, they are chosen to represent rooms. It is also convenient for further analysis and process because the subordinate relation between doors and the room is definite. It is worth mentioning that some complicated rooms such as elevators or rooms with an internal compartment should be treated specially. For the former, in order to reduce the difficulty of process, the elevators and escalators are abstracted as rooms with doors, presented as nodes. While for the latter, rooms are divided for the cases of with and without 
compartments. Similar to single-floor route model, the route model in a room which can be thought as a sub one.

4.1.7 Compartments connection: A complete single-floor route requires not only topological route, but also the compartments should be added to the route model. Generally, rooms are represented with central point. However, there may be more than one doors for a room, especially some business towers with complicated internal structures. The route solution calculated with room's central point may not be the most optimal in this case. Since doors are the connection between rooms and the corridor, they are chosen to represent rooms. It is also convenient for further analysis and process because the subordinate relation between doors and the room is definite. It is worth mentioning that some complicated rooms such as elevators or rooms with an internal compartment should be treated specially. For the former, in order to reduce the difficulty of process, the elevators and escalators are abstracted as rooms with doors, presented as nodes. While for the latter, rooms are divided for the cases of with and without compartments. Similar to single-floor route model, the route model in a room which can be thought as a sub one.

4.1.8 Single floor route model: According to above algorithms about corridor route extraction and compartments connection, the sing floor route model can be constructed conveniently, as Figure 7 shown.

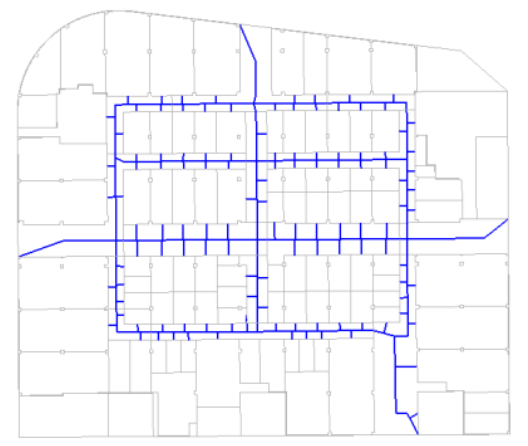

Figure 7 Route modelling for single floor

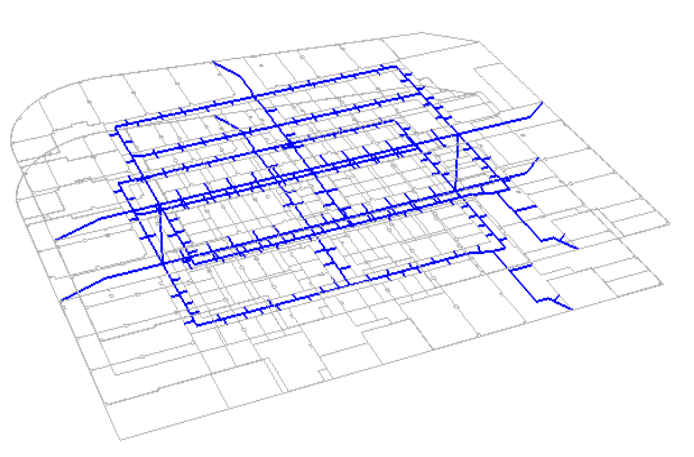

Figure 8 The result of building indoor route model

\subsection{Multi-floor route model construction}

The other route models can be created based on the above method of single floor route model construction, and the whole indoor $3 \mathrm{~d}$ route model will be constructed through connecting all floors according to the real special relationship, specifically the connection of stairs, element, etc. Figure 8 shows the result of building indoor 3D route model.

\section{IMPLEMENTATION AND EXPERIMENT}

In order to validate the method in this paper, a shopping mall called "Longjiang New City Plaza" in Nanjing is chosen as a case of study, and a prototype system has been designed and developed.

\subsection{Experiment area}

Longjiang New City Plaza covers an area of 126,000 square meters, construction area of 28 million square meters, with nearly 400 brands of specialty stores. It is a shopping, dining, entertainment, leisure and sports, culture, social, office, living spaces integrated business center. So, it is useful for practical applications to select this area for experiment.

\subsection{Experiment design}

Figure 9 shows the process of route modelling for single floor in Longjiang New City Plaza on the basis of the algorithms designed in 3.1 .

Figure 10 shows the result of route modelling for multi-floor.
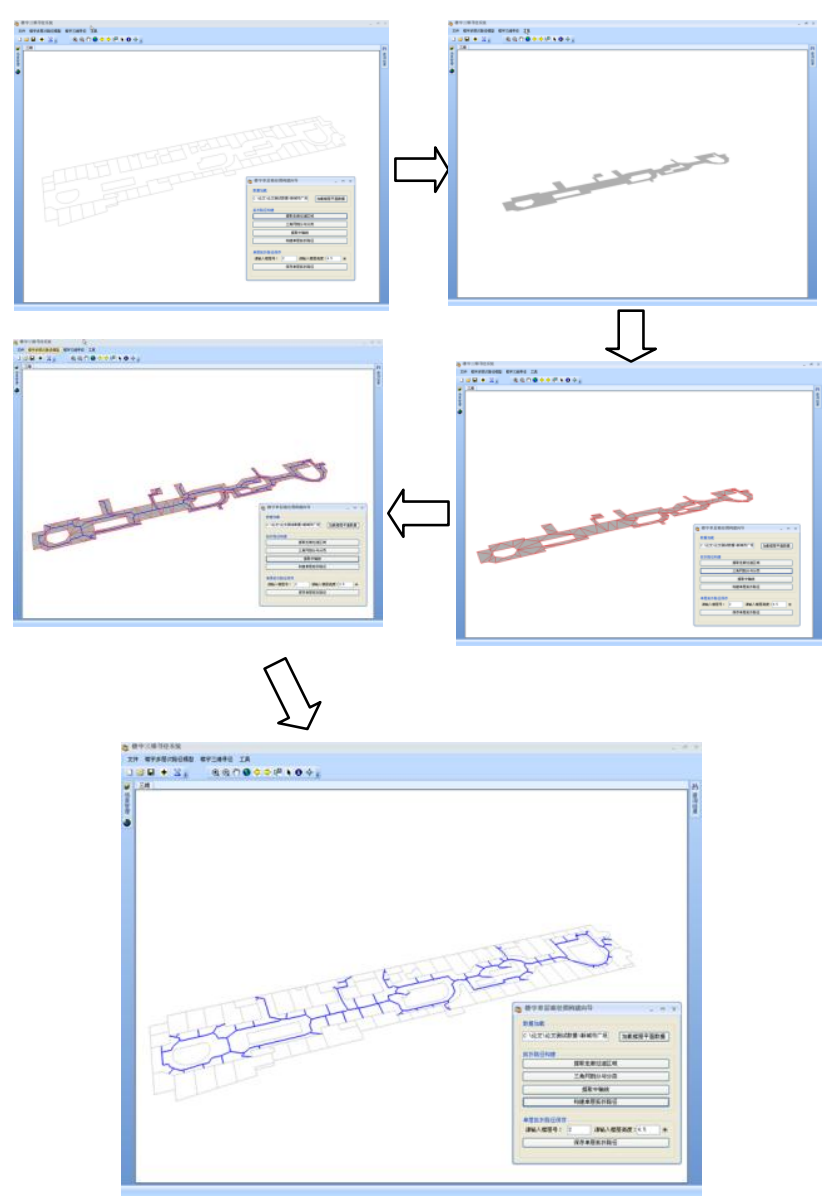

Figure 9 The result of route modelling for single floor in Longjiang New City Plaza 


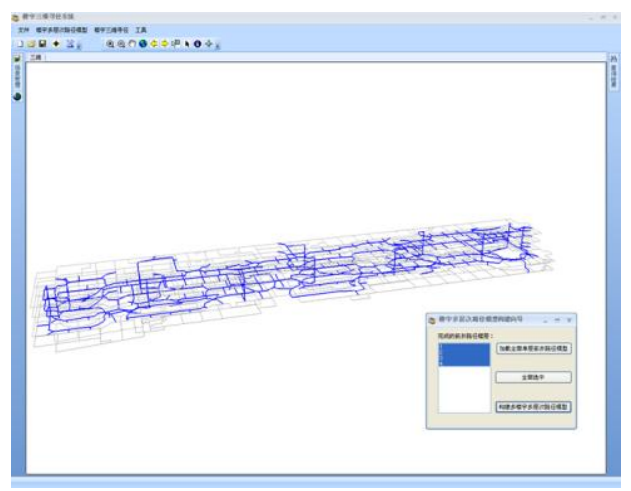

Figure 10 The result of route modelling for multi-floor in Longjiang New City Plaza

At last, the evacuation simulation was made using the model in this paper for validating the validity (Predtechenskii, 1978).

The calculation parameters of $\mathrm{CO}$ are set according to the smoke sensor. As shown in Figure, the smoke sensor detects the parameters of $\mathrm{CO}$ is up to 0.8 , which means the dense of smoke will imperil the people's life in this area. The evacuation path should be regenerated because the equivalent length of the path need to be recalculated on the basis of $\mathrm{CO}$ parameter, eg. Figure 11 .
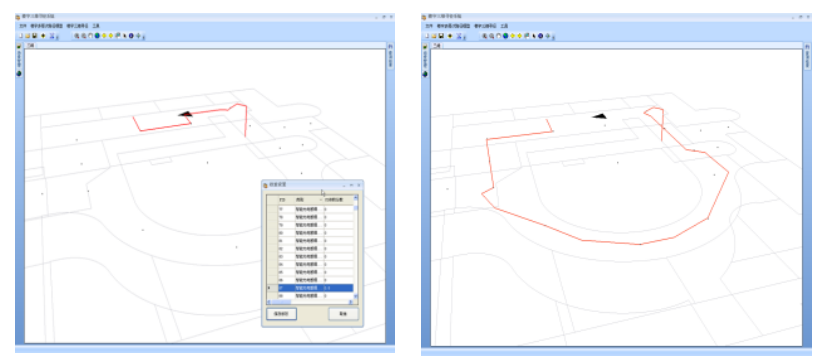

Figure 11 Seneor alerting and route regenerating

Emergency evacuation for a floor should consider that the people in all compartments evacuate with control and the best evacuation route and theoretical evacuation time. Figure 16 shows the evacuation simulations in Longjiang New City Plaza. There are 8 fire stairs for people to evacuate to a safer place outside. The width of stair is 2.2 meters, while the width of emergency exit is 1.8 meters. The people flow coefficients are assumed $0.62(\mathrm{per} / \mathrm{m} \cdot \mathrm{s})$ and $1.10(\mathrm{per} / \mathrm{m} \bullet \mathrm{s})$.

The business area of the third floor is 3919 square meters, and the number of evacuation people is 3919 (per) $* 0.77=3018$ (per) according to the conversion factors from "Code for design of store buildings".

5.2.1 Corridors evacuation time: According to the route model, the max horizontal distance of corridor evacuation is 28.47 meters. So,

$$
t_{1}=L / V=28.47 / 0.35 \approx 81.34(s)
$$

\subsubsection{Stairs evacuation time:}

$$
t_{2}=\frac{P}{W_{S} F_{S}}=\frac{3018}{2.2 * 8 * 0.62} \approx 276.58(s)
$$

\subsubsection{Security exits evacuation time:}

$$
t_{3}=\frac{P}{W_{e} F_{e}}=\frac{3018}{1.8 * 8 * 1.1} \approx 190.53(s)
$$

\subsubsection{Overall evacuation time}

$$
T=t_{1}+t_{2}+t_{3} \approx 548.45(s)
$$

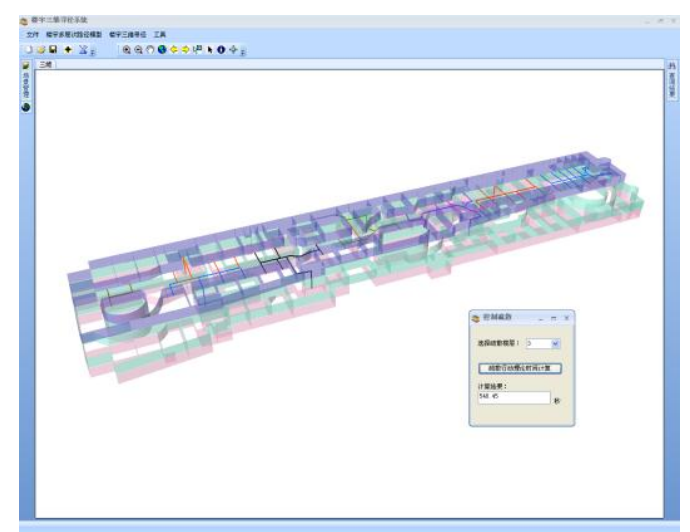

Figure 12 The result of evacuation route construction in Longjiang New City Plaza

The different colors in Figure 12 indicate evacuation routes in different areas.

\section{CONCLUSION}

An effective method of indoor three-dimensional route retrieving in the building is introduced in this paper, and the detailed modelling process is discussed. The algorithm takes advantage of estate special data, and is realized constrained three-dimensional route model automatically without user intervention. This study provides good application value, which improves the efficiency and precision of route modelling, also the geometry and semantic information is saved. Also, because of the weighted edges, route planning can be calculated according to the weights easily. Experiment show that the indoor three-dimensional route modelling method based on estate spatial data in this paper can support indoor route planning and evacuation route design very well, and the result can also be displayed in a 3D scene intuitively. With the development of LBS, more and more indoor applications were demanded in the further, and this study has good application prospects.

\section{REFERENCES}

Huang, Y., Du, J., \& Chen, H. (2010). Construct threedimensional route from blueprint image of building. Beijing Jiaotong Daxue Xuebao(Journal of Beijing Jiaotong University), 34(2), 79-83.

Krieg-Brückner, B., Frese, U., Lüttich, K., Mandel, C., Mossakowski, T., \& Ross, R. J. (2005). Specification of an ontology for route graphs. In Spatial Cognition IV. Reasoning, Action, Interaction (pp. 390-412). Springer Berlin Heidelberg.

Stevens, M., \& Choi, J. (2006). CAD Data conversion to a node-relation structure for 3D sub-unit topological representation. Journal of the Korean Geographical Society, 41(2), 188-194. 
Stoffel, E. P., Lorenz, B., \& Ohlbach, H. J. (2007). Towards a semantic spatial model for pedestrian indoor navigation. In Advances in Conceptual Modeling-Foundations and Applications (pp. 328-337). Springer Berlin Heidelberg.

Preparatat, F. P., \& Shamos, M. I. (1985). Computational geometry: an introduction.

Predtechenskii, V. M., \& Milinskiǐ, A. I. (1978). Planning for foot traffic flow in buildings. National Bureau of Standards, US Department of Commerce, and the National Science Foundation, Washington, DC.

Schadschneider, A., Klingsch, W., Klüpfel, H., Kretz, T., Rogsch, C., \& Seyfried, A. (2009). Evacuation dynamics: Empirical results, modeling and applications. In Encyclopedia of complexity and systems science (pp. 3142-3176). Springer New York. 